\title{
Multi-Objective Evolving Neural Network supporting SDR Modulations Management
}

\author{
Valeria Loscrí, Pasquale Pace and Rosario Surace \\ DIMES - Department of Informatics, Modeling, Electronics and System Engineering \\ University of Calabria, Cosenza, ITALY \\ Email: \{vloscri, ppace, rsurace\}@dimes.unical.it
}

\begin{abstract}
This paper proposes a distributed Neural/Genetic algorithm able to compute both the more suitable positioning and transmission modulation schemes for fixed/mobile wireless nodes equipped with software defined radio abilities. Devices considered in this work are able to move towards new positions by applying the concept of controlled mobility. The selection of the more suitable modulation scheme is realized through the SDR (Software Defined Radio) paradigm. The synergistic combination of controlled mobility and SDR in a totally distributed way, allows to obtain a high degree of self-configurability; moreover, the extreme adaptability to the network conditions and application level constraints in terms of coverage and guaranteed connectivity, make the proposed approach well suited for quite different communication scenarios such as classical monitoring or disaster recovery. The obtained results, validated throughout an intensive simulation campaign, show how the controlled mobility paradigm applied to the wireless devices and the intrinsic re-configuring SDR capabilities, increase the performance of the network both in terms of coverage and connectivity respect to other algorithms.
\end{abstract}

Index Terms-Self-Organizing Networks, SDR, Controlled Mobility, Neural Network, Genetic Algorithm.

\section{INTRODUCTION}

In the last few years, the wireless networks research community has investigated the idea of self-organizing devices capable to self-configure and adapt their behaviors to the current environment without human intervention. Starting from this general communication trend, we consider to deploy a communication network in an area in which several wireless devices, scattered all around, can self-configure to carry out a common task by providing connectivity towards a sink node placed in the center of the area.

Within this general communication scenario, the selfconfiguring characteristics supported by the wireless nodes are: (i) transmitting/receiving data over different links by using different modulation schemes implemented throughout a specific SDR architecture (ii) changing their position within a specific area in order to find the best place to optimize network parameters and application goals such as coverage and connectivity with guaranteed QoS also minimizing the energy consumption due to the transmission.

In our vision, the controlled mobility paradigm coupled with the software defined radio capabilities of such novel wireless devices can improve the performances of self-organized networks able to self-adjust their parameters to handle very unlike communication scenarios. According to this vision, the paper proposes a distributed Neural/Genetic algorithm to compute the final nodes positions and the modulation schemes for each transmitter/receiver pair in order to guarantee the agreed QoS level. In particular, the ability to automatically select, the correct modulation scheme used in an unknown received signal is a major advantage in a wireless network that can be achieved by using a SDR architecture [1]. In this way, as a channel capacity varies, modulation scheme switching enables the baud rate to be increased or decreased maximizing channel capacity usage. Furthermore, within the analyzed framework, we will show how the SDR capabilities supported by the wireless node, coupled with the controlled mobility functionality, can improve the system performances in terms of connectivity by making these mobile SDR nodes very suitable for communication scenarios in which the requirements on constrained QoS connectivity, are more stringent respect to the ones on the maximum coverage.

An example of such communication scenario could be the case of a disaster area where the communication between the survivors and the rescue teams has to guarantee a good quality level [2]; on the other side, a communication scenario related to applications such as pollution monitoring or fire detection only need a high coverage degree that can be achieved by taking advantages only from the mobility of the nodes without using the SDR capabilities.

The rest of the paper is organized as follows: Section II describes the devices involved in the communication scenario highlighting their hardware and software capabilities. Section III presents the Neural/Genetic algorithm able to compute the best positioning for the wireless nodes to satisfy the constraints imposed by the specific communication scenario. Section IV discusses the obtained results and the goodness of the proposed approach in terms of extreme adaptability whilst the conclusions and future research directions have been drawn in Section V.

\section{SDR DEVICES}

In this work we consider a communication scenario in which different devices can be used to form a self-evolving wireless network in order to reach several goals such as coverage increase, high data rate and connectivity also supporting different applications. In particular, we suppose to have both simple mobile or fixed sensor nodes equipped with a wireless 


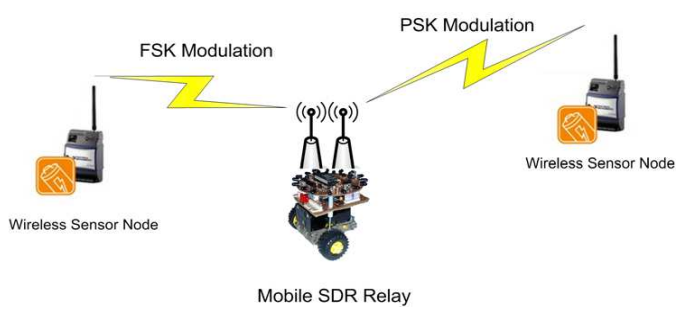

Fig. 1. Mobile relay devices supporting SDR capabilities

IEEE 802.15.4/ZigBee compliant RF transceiver and more complex mobile devices with a high processing capability, able to support the SDR functionality to dynamically change the modulation scheme between different transmitter/receiver pairs within the same communication path as shown in Figure 1. In addition, we assume our wireless devices are equipped with a GPS module and a specific software application that make them able to exchange positions between two neighbor nodes in order to perform a specific positioning strategy as described in the next section.

The programmable architecture of SDR promotes a flexible implementation of several modulation schemes (i.e. MFSK, MPSK, MQAM). This flexibility also translates into adaptivity to optimize different network performance indexes such as coverage, degree of connectivity or throughput of a wireless network operating under varying channel conditions. In this context, since these more complex devices are equipped with SDR functionalities, they can easily work as relay nodes in a multi-hop communication scenario by dynamically adapting different modulation schemes between the receiving and transmitting phases with the aim of optimizing network performances such as BER (Bit Error Rate), energy consumption and overall coverage.

It is well known that channel modulation has a huge impact on the quality of the sent information measured in terms of BER and on software/hardware complexity of the receiver that dominates the overall complexity of the SDR architecture because it is typically four times more complex than the transmitter in terms of MIPS (Million Instructions per Second) required to implement the baseband and the IF (Intermediate Frequency) processing in the software; furthermore, digital modulation/demodulation techniques need specific channel waveform coherence, coding/decoding and spreading/dispreading of the radio spectrum [3]. In particular, the bit error probability is a function of channel modulation; thus, working with three main digital modulation types (i.e. MFSK, MPSK, MQAM) having different modulation orders $(M=2,4,8,16)$, a radio channel with better quality can be assigned to a larger number of bits and a higher order modulation, whereas a channel with poorer quality has to be assigned fewer bits or even no bit when the channel quality is too bad. Moreover, to guarantee a certain BER value, the modulation schemes should be changed according to the channel quality experienced by the nodes and the distance variation between nodes due to the mobility.

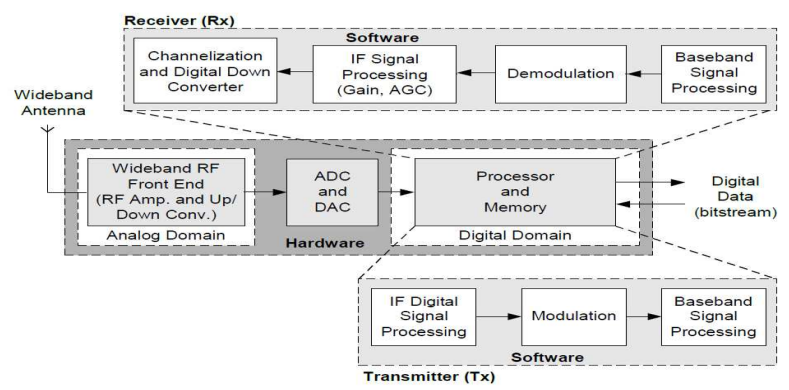

Fig. 2. SDR architecture for Relay Node [4].

In the last few years, the SDR paradigm is becoming more attractive and feasible thanks to the development of open-source software toolkit such as GNU radio [5] and hardware devices such as Universal Software Radio Peripheral (USRP) [6]; therefore, several modulation/demodulation software blocks can be developed within the generic SDR architecture [4] for both transmitter and receiver (see Figure 2 ) allowing the design of new and more powerful devices well suited to support dynamic modulation changing and adaptation strategies proposed in this work.

\section{Neural Network And Genetic Algorithm}

The main objective of the algorithms considered in this work is the computation, in a totally distributed way, of a suitable position for wireless devices to satisfy some specific network requirements. Since each node considers its own neural network to move and the genetic algorithm to perform a new solution that better fits with the objective function, the computation of the new nodes' position can be only based on local information. For this reason both algorithms are performed in a distributed way: a node computes the neural algorithm by knowing the positions of its neighborhood and the genetic algorithm manages its own genes without global information.

Specifically, the two objectives considered in this work, namely, the coverage area and the number of sensor devices having a path toward a sink with a certain quality of service are in contrast to each other.

Therefore we have to design a wise strategy able to take into account both the requirements in a dynamic and reconfigurable fashion. The proposed algorithm is mainly based on the approach presented in [7] and it consists of two parts: a neural component and a genetic algorithm.

\section{A. The Neural Network}

The behavior of each wireless node is controlled through a neural network. It is fully connected, recurrent and timediscrete. The neural network consists in input, output and hidden neurons. Inputs are subdivided as follows:

- 4 inputs to detect overlapping of sensing zone with neighborhood' sensing zone (1 for each direction);

- 4 inputs to detect missing of sink connection (1 for each direction); 
- 1 to detect nodes in the same position.

The output is the new position. Each neuron "actives" a real-valued function and a time-varying real-valued connection with every other neuron of the network to map input (n-dim) in output (m-dim). We indicate with $o u t_{j}$ the output of neuron $j$ towards all other neurons of the network. The output of neuron $j$ is computed as shown in Equation (1).

$$
\text { out }_{j}(k)=F\left(\sum_{i \in N} w_{i j} \cdot \text { out }_{i}(k-1)+b_{j}\right)
$$

where $N$ is the set of neurons, $w_{i j}$ is the weight of the connection between neuron $i$ and neuron $j$ and $b_{j}$ is the bias of neuron $j$. Weights can produce both excitatory or inhibitory effect. The activation function $F$ is the following linear threshold function:

$$
F(x)=\left\{\begin{array}{llc}
-1.0 & \text { if } & x \leq-1.0 \\
x & \text { if } & -1.0<x<1.0 \\
1.0 & \text { if } & x \geq 1.0
\end{array}\right.
$$

For each node the output of the neural network is given from the two output neurons and it is consists of two real numbers that vary in the range $[1,-1]$, as it is clear from (2). Based on these two values, the node chooses the action to do. Assuming a square field of $n \times n$ cells, the node can move in one of the four allowed directions or remain in the current cell.

\section{B. The Genetic Algorithm}

A conventional and real-value Genetic Algorithm (GA) is used in the training phase of the Neural Algorithm. The genes are associated with the connections weights between each couples of neurons and the bias of each neuron. Through the typical operators of genetic approaches (i.e. crossover, mutation and selection), different weights to the neurons in the next generations will be assigned. Of course, the chromosome selected for the next generations is the one which has the best value of the fitness function. In our work, we consider a biobjective functions in order to: 1) maximize the coverage; 2) maximize the number of nodes connected to the sink either in a direct fashion or through a multi-hop path. The fitness function can be written as follows:

$$
\text { fitness }=\alpha * \text { Coverage }+\beta * Q o S_{\text {connectivity }}
$$

where

$$
\text { Coverage }=\frac{\text { covered_area }}{\text { whole_area }}
$$

and

$$
Q o S_{\text {connectivity }}=\frac{\# \text { nodes_connected_to_the_sink }}{\text { \#total_nodes }}
$$

where $\alpha$ and $\beta$ are weights that take into account the priority of the objective to be reached.

The term \#nodes_connected_to_the_sink represents the number of nodes "connected" to the sink, namely nodes that are able to reach the sink directly or for which exists a multihop path [8] throughout links that are able to deliver data by guaranteeing a certain value of BER given as an input constraint. To the follow we show the pseudo-code of the Neural/Genetic Algorithm.

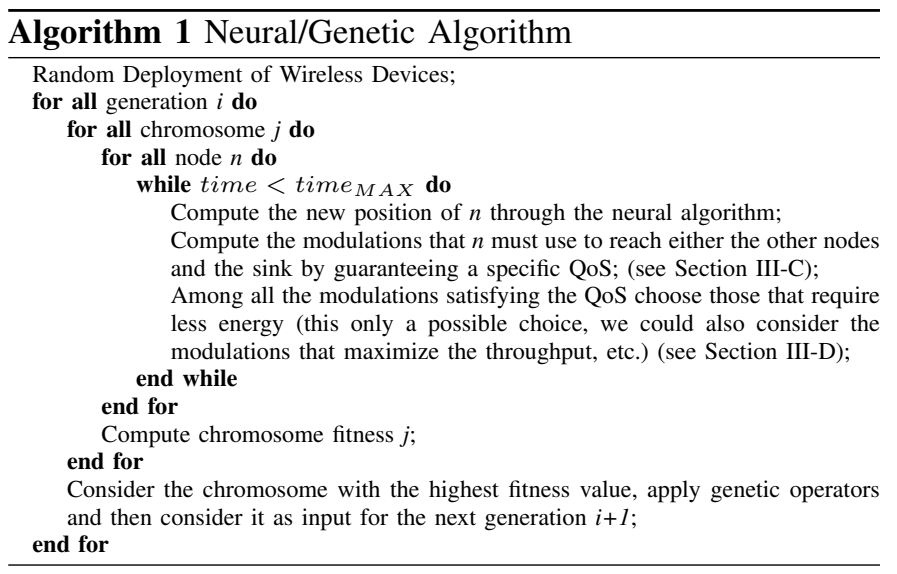

\section{Supported Connectivities and Communication Complexity}

As already explained, each node has a set of possible modulations that can be "used" for data transmission by guaranteeing different connectivity levels. In this work, we figure out two different types of connectivities:

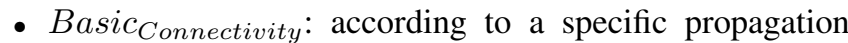
model, it is possible to compute the maximum distance at which a node $n_{1}$ is able to transmit, that is the transmitting radius. If a node $n_{2}$ is inside the area delimited by the circle with radius equal to the transmitting radius of the node $n_{1}$, then $n_{1}$ and $n_{2}$ are connected. In this work we consider the propagation model as defined in [9]:

$$
P L_{\text {generic }}=\left(\frac{4 \pi d_{0}}{\lambda}\right)^{2} \cdot\left(\frac{d}{d_{0}}\right)^{\gamma}+\chi
$$

where $d$ is the distance between the $T x$ and the $R x, \gamma$ is the path loss exponent, $\lambda$ is the wavelength, $\chi$ is the shadowing effect value (neglectable) and $d_{0}$ is the critical distance.

- QoS Connectivity: each node $n$ has a neighborhood. For every neighbor, the node $n$ computes the BER value on the specific link by considering the different available modulations. The node $n$ excludes all the modulations that do not respect the BER required as constraint in input. In practice, in this way the node $n$ has a set of neighbors and every link from $n$ to the neighbor meets the QoS constraint in terms of BER.

For each iteration of the algorithm and for every node, both the Basic Connectivity and the $Q o S_{\text {Connectivity }}$ are computed.

Since the proposed algorithm is based on local communication, a node only needs to know the position of its neighboring nodes to make a movement. According to [7], after each nodes movement, an update on the nodes position is broadcasted through a constant size message containing the node identifier (Id) and the node position $(x, y)$, therefore the message size is 
in $O(1)$. As a consequence, considering a constant value for the number of time steps given as an input parameter, nodes will update their positions and broadcast their new information at each time step; this leads to a linear message sending complexity of $O(n)$ where $n$ is the number of nodes within the network.

\section{Transmitted Energy Computation}

In order to save energy for the transmission always guaranteeing the required quality of service degree, it is necessary to choose the less power hungry modulation scheme within the set of the the most common modulation schemes available in real devices. To this aim, the energy spent per information bit $[J]$ can be computed as follows [11]:

- For both MQAM and MPSK, by considering a signal bandwidth equal to $B[H z]$ and, by assuming a sample time $T_{s} \approx 1 / B$ [12], we can write:

$$
\begin{aligned}
E_{\text {infBit }} \approx & \frac{(1+\delta) \cdot S N R \cdot N_{0} \cdot N_{f} \cdot G_{d}}{R} \\
& +\frac{P_{c}}{R \cdot B}+\frac{P_{t r} \cdot T_{t r}}{L}
\end{aligned}
$$

where $\delta=\xi / \eta-1, \xi$ is the peak-to-average power ratio (PAPR) of the signal depending on the specific modulation, constellation size and shape ${ }^{1}$ whereas $\eta$ is the efficiency of PA drain chosen equal to 0.35 as typical value of class A power amplifiers [12]. $P_{t r}$ and $T_{t r}$ are the consumed power and the time spent in transient mode respectively whilst $L$ is the total number of information bits. The $S N R=\frac{P_{r x} \cdot T_{s}}{N_{0} \cdot N_{f}}$, where $P_{r x}$ is the received signal power, $N_{0} / 2$ is the power spectral density of the noise and $N_{f}$ is the receiver noise figure. Moreover, by assuming a general path-loss model, the value of $G_{d}$ can be computed according to the Equation (6) and it is equal to $G_{d}=\left(\frac{4 \pi d_{0}}{\lambda}\right)^{2} \cdot\left(\frac{d}{d_{0}}\right)^{\gamma}$. Finally, the term $P_{c}$ represents the circuit power (i.e. $211[\mathrm{~mW}]$ for both MQAM and MPSK) and the term $R$ is the transmitting rate computed for each constellation by using the cutoff curves.

- For the MFSK modulation, by considering a noncoherent detection, the well known relation $M=2 T_{S} B$ [10] allows to derive $T_{S}=M / 2 B$, therefore the energy for the transmission will be equal to:

$$
\begin{aligned}
E_{\text {infBit }}= & \frac{(1+\delta) \cdot S N R \cdot N_{0} \cdot N_{f} \cdot G_{d}}{R} \\
& +\frac{P_{c} \cdot M}{2 \cdot R \cdot B}+\frac{P_{t r} \cdot T_{t r}}{L}
\end{aligned}
$$

where $\eta=0.75$ is the typical value for class $\mathrm{B}$ or even greater $(\mathrm{C}, \mathrm{D}$, or $\mathrm{E})$ power amplifiers [12], $\xi=1$ according to [11] and $P_{c}=165.3[\mathrm{~mW}]$ for general MFSK modulation schemes.

\footnotetext{
${ }^{1} \xi=3 \cdot \frac{(\sqrt{M}-1)}{\sqrt{M}+1}$ for square constellations whereas $M$ is the constellation size, while it assumes a value among those shown in Tab. 1 of the work [11] for cross-shaped constellations.
}

\section{Simulations And Results}

The proposed scheme is evaluated by simulations using $\mathrm{FREVO}^{2}$, an open source framework for evolutionary design. We took into account a $40 \times 40$ cells field, where 64 nodes are placed in a random way according to an uniform distribution. We considered one cell and one time step as discrete units of space and time, respectively. Also the sensing radius of the nodes is $r=2[$ cells] and it expresses the number of cells that nodes are able to cover in each of the four main direction (north, south, east and west). For the neural network, we use 9 input neurons, 2 hidden neurons and 2 output neurons. For the genetic algorithm, we use 300 chromosomes and 100 generations. All the results have been averaged over 10 different runs to respect a confidence interval of $95 \%$.

In order to conduct a quite realistic analysis on the energy consumption, we chose to set transmitting power $(12 \mathrm{dBm})$ and receiver sensitivity $(-80 \mathrm{dBm})$ of our devices by referring to an off-the-shelf Bluetooth module made by Roving Networks [13]. Table I summarizes all the other simulation parameters used.

TABLE I

VALUES OF THE RELEVANT PARAMETERS USED FOR THE SIMULATIONS

\begin{tabular}{cc}
\hline \hline Device Parameters & \\
\hline Power spent in transient mode $\left(P_{t r}\right)$ & $100[\mathrm{~mW}]$ \\
Time spent in transient mode $\left(T_{t r}\right)$ & $5[\mu \mathrm{s}]$ \\
Wavelength $(\lambda)$ & $0.125[\mathrm{~m}]$ \\
Information bits $(L)$ & $1000[\mathrm{bits}]$ \\
Receiver noise figure $\left(N_{f}\right)$ & 10 \\
Bandwidth $(B)$ & $10[\mathrm{kHz}]$ \\
\hline \hline Scenario Parameters & \\
\hline Path Loss Exponent $(\gamma)$ & 1.8 \\
Critical distance $\left(d_{0}\right)$ & $\left.1 \mathrm{~m}^{-15}\right]$ \\
PSD of the noise $\left(N_{0} / 2\right)$ & $\left.10^{-3} / \mathrm{Hz}\right]$ \\
Bit Error Rate $\left(B E R_{t h r e s h o l d}\right)$ & 100 \\
Maximum number of time steps & $15 \%$ \\
\hline \hline Genetic Algorithm Parameters & $45 \%$ \\
$\%$ of elite selection $(e)$ & $30 \%$ \\
$\%$ of mutation $(m u)$ & $5 \%$ \\
$\%$ of crossover $(c)$ & $5 \%$ \\
$\%$ of randomly created offsprings $\left(\right.$ off $\left._{c}\right)$ & \\
\hline \hline
\end{tabular}

\section{A. Fixed nodes analysis supporting SDR}

In this section we show the results obtained by supporting the SDR capabilities; in this context the communication devices are all fixed but they can autonomously decide to use one of three different modulation schemes (MFSK, MPSK, MQAM) with three different symbol levels $M$ (4, $8,16)$ thus the set of possible choices is extended to nine. However, since the mobility support is out of the scope of this first reference simulation scenario, the Neural/Genetic algorithm described in section III cannot be executed every

\footnotetext{
${ }^{2}$ http://www.frevotool.tk
} 
new generation and the result, in terms of more suitable modulation schemes in agreement with the desired QoS, is always the same representing the reference benchmark point for the next analysis in which the mobility of the nodes allows to achieve better performances. By using the SDR capabilities it is possible to increase the $\mathrm{QoS}_{\text {connectivity }}$ because every node can have more communication choices to create a link with a neighbor that supports at least one of its known modulation schemes; nevertheless, the performance in terms of Coverage cannot take advantages from the SDR capabilities because the devices cannot change their positions as already explained. Table II summarizes the obtained results over 1000 simulation runs, also specifying the percentage of nodes that have chosen any specific modulation scheme and the average energy consumption for the transmission. It is worth to note that in the simulated scenario few modulation schemes such as 4-8-PSK have never been chosen due to the worst performance in terms of BER and to the higher energy consumption.

TABLE II

FIXED NODES ANALYSIS SUPPORTING OR NOT SUPPORTING SDR CAPABILITIES

\begin{tabular}{ccc}
\hline \hline Output Parameters & Without SDR & With SDR \\
\hline Coverage & $61.57 \%$ & $61.47 \%$ \\
QoS connectivity $_{\text {Energy per Information Bit }}$ & $2.40 \cdot 10^{-5}[\mathrm{~J}]$ & $2.75 \cdot 10^{-5}[\mathrm{~J}]$ \\
Nodes choosing 4-FSK & $12.59 \%$ & $0 \%$ \\
Nodes choosing 8-FSK & $28.34 \%$ & $38.57 \%$ \\
Nodes choosing 16-FSK & $34.82 \%$ & $43.92 \%$ \\
Nodes choosing 4-PSK & $0 \%$ & $0 \%$ \\
Nodes choosing 8-PSK & $0 \%$ & $0 \%$ \\
Nodes choosing 16-PSK & $10.80 \%$ & $10.94 \%$ \\
Nodes choosing 4-QAM & $0 \%$ & $6.57 \%$ \\
Nodes choosing 8-QAM & $0 \%$ & $0 \%$ \\
Nodes choosing 16-QAM & $13.45 \%$ & $0 \%$ \\
\hline \hline
\end{tabular}

\section{B. Mobile nodes analysis supporting SDR}

In this section we show how controlled mobility can be efficiently exploited to reach better configurations both in terms of Coverage and $Q o S_{\text {connectivity. In Figures } 3 \text { we show results }}$ obtained when all nodes are equipped with motion capabilities but they are not able to select, in a dynamic fashion, the most suitable modulation. Specifically, all nodes will support only a specific modulation scheme in a random way, by keeping the percentage of nodes that choose a certain modulation equal for all the modulation schemes. In this specific case, the $33.33 \%$ of nodes will support FSK or QAM or PSK modulation. Of course, in order to obtain reliable results we averaged them in order to have a confidential interval of $95 \%$. In this scenario, all nodes will move in a distributed fashion towards novel positions computed through the neural network by considering a genetic approach during the training phase as explained in Section III. It is worth to notice that in the configuration where nodes are not able to move, Coverage cannot be improved and nodes are only allowed to choose a better modulation in order to improve $Q o S_{\text {connectivity. When nodes are able }}$ to move, better configurations in terms of both Coverage and $Q o S_{\text {connectivity }}$ are obtained. For $\alpha$ values ranging from 0.5 to 1 , after 20 Generations the nodes are able to reach a percentage of coverage higher than $90 \%$. Concerning the $Q o S_{\text {connectivity }}$ index, it is increased from $12.2 \%$ to $35 \%$ after $\approx 35$ Generations by tuning the connectivity parameter $(\beta)$ with higher values, 0.75 and 1 . Unfortunately, if we observe curves related to Coverage and QoS connectivity in a crossway, we notice as controlled mobility is a valid tool to improve performance of the system, but coverage and connectivity are opposite goals, and then controlled mobility is able to generate configuration that "answer" in an effective way to the setting of $\alpha$ and $\beta$, but it is not able to handle the opposition of those two QoS requirements. In fact, in Figure 3 (a), when the value of $\alpha$ is chosen equal to 0.25 , the network is not able to reach a degree of coverage higher than $\approx 24 \%$, and this is the case (see Figure 3 (b)), connectivity reaches $\approx 42 \%$. By considering an additional freedom degree consisting into the possibility to set the most suitable modulation, the overall performance of the system are improved as shown in Figure 4 (a) and (b). A strange effect of the dynamic modulation setting possibility occurs when $\alpha$ and $\beta$ are both set equal to 0.5 . In this case (see Figure 4 (a)), coverage reached is smaller than in the previous case, but it is worth to analyze this behavior in combination with the connectivity value. In fact, in Figure 4 (b), in correspondence of the same $\alpha$ and $\beta$ values, we are able to obtain a connectivity degree higher than $93 \%$ after very few Generations. On the other hand, the system gives an answer matching the interest we express with the $\alpha$ value, since we set $\alpha$ equal to 0.5 . When the $\alpha$ value is higher than 0.5 , the system takes properly into account this, and coverage increases. From this analysis, we can argue that by considering in a similar way the importance of both $\alpha$ and $\beta$ parameters, the system will behave in a very effective fashion guaranteeing a very high level of $Q o S_{\text {connectivity }}$ and a good degree of Coverage. These results are also confirmed by the Fitness curves shown in Figures 3 (c) and 4 (c) respectively. In fact, we can observe as Fitness improves by reaching very high values after a few number of generations when the weight associated with connectivity is the highest possible $(\alpha=0$ and $\beta=1$ ). In respect of the case in which nodes are only equipped with motion capabilities, SDR mobile nodes are able to react to the connectivity requests of the networks. Moreover, in all the studied cases we can notice an improvement of the Fitness except when coverage is considered as a kind of high priority (i.e. $\alpha=0.75$ or $\alpha=1$ ) making the Fitness trend similar to the case with no-SDR mobile nodes. As main conclusion of this simulation campaign, we can argue that, by correctly tuning the $\alpha$ and $\beta$ weights of the Fitness function, the wireless network consisting of self-configuring SDR devices can dynamically react in order to face different communication scenarios by favoring, from time to time, the Coverage, the $Q o S_{\text {connectivity }}$ or both. 


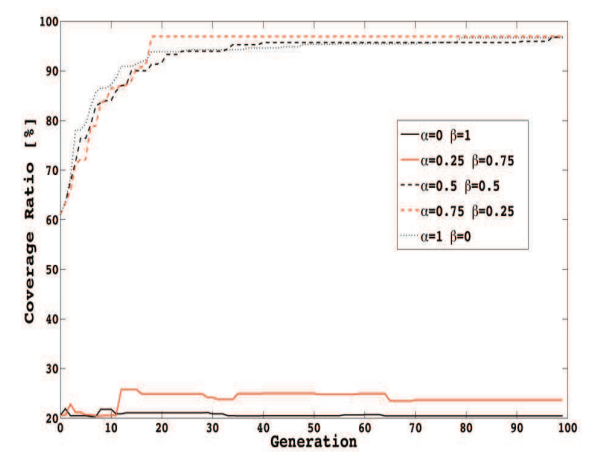

(a)

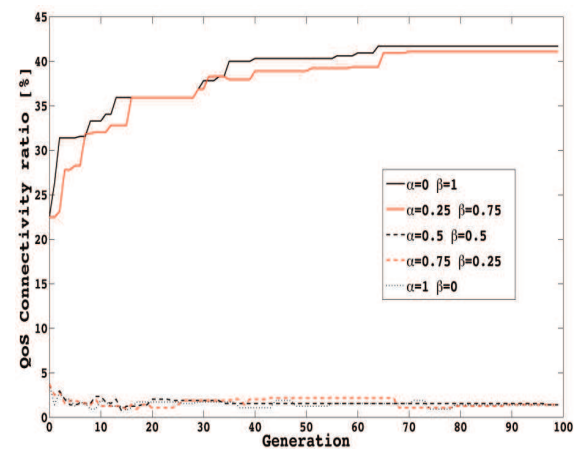

(b)

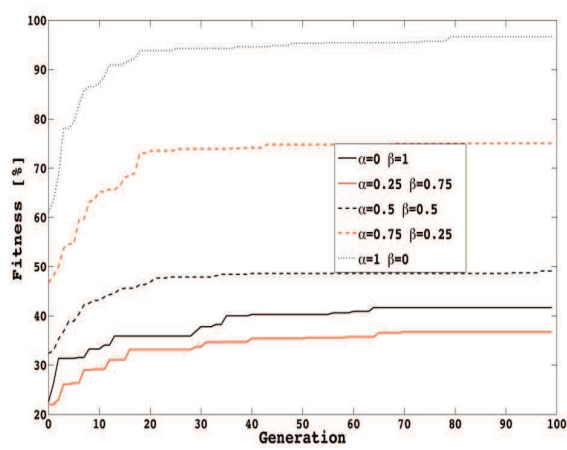

(c)

Fig. 3. Neural/Genetic algorithm supporting mobility without SDR capabilities: (a) Coverage, (b) QoS connectivity $_{\text {, (c) Fitness }}$

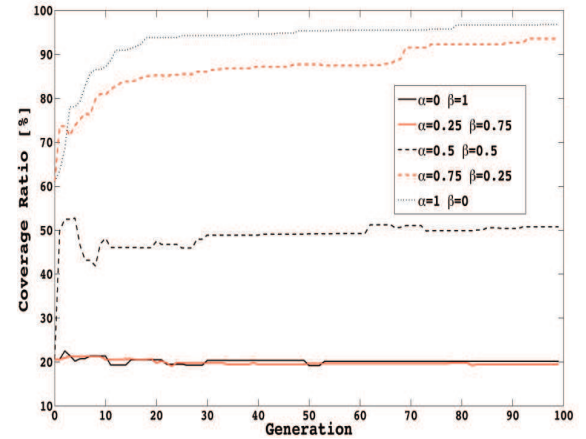

(a)

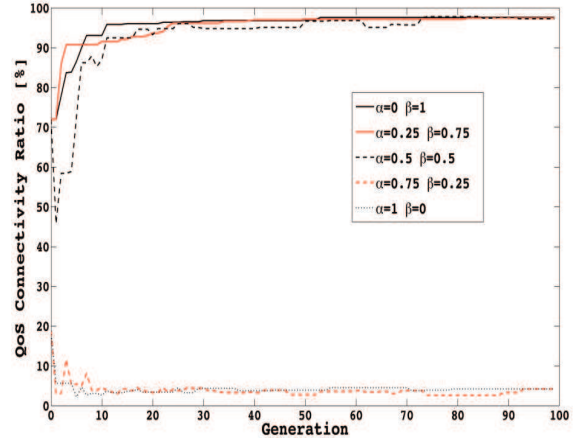

(b)

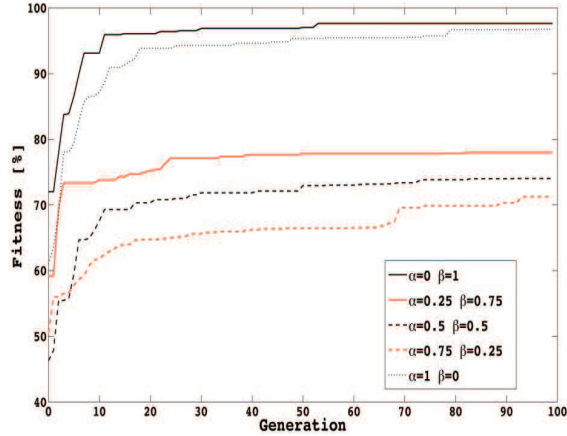

(c)

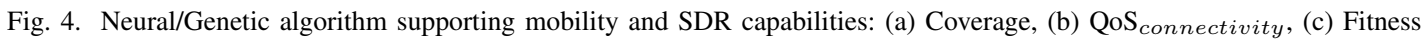

\section{CONCLUSIONS}

In this paper we considered SDR mobile nodes able to move towards most suitable positions and to select the best modulation scheme in order to both improve the coverage of a specific area and the connectivity to a sink node. All nodes run an algorithm based on a totally distributed Neural/Genetic approach by using only local information. Simulation results showed as intrinsic re-configuring SDR capabilities allow to reach better performance of the network evaluated in terms of both coverage and connectivity. Moreover, simulations allowed to conclude that controlled mobility can be very effective in terms of coverage, but when two opposite requirements such as coverage and connectivity are considered, the combination of SDR and controlled mobility features, allow to reach very good results. As future work, it is necessary to analyze how the amount of SDR devices impact on the networks performance because SDR equipment could be prohibitive in terms of cost.

\section{ACKNOWLEDGMENT}

This work has been carried out under the framework of STEMNet, PRIN-National Italian Project \#H21J11000050001, financed by the Italian Ministry of University and Research.

\section{REFERENCES}

[1] B. B. Godbole and D. S. Aldar, "Performance Improvement by Changing Modulation Methods for Software Defined Radios", International Journal of Advanced Computer Science and Applications, 1(6), Dec. 2010.
[2] P. Pace and G. Aloi, "Disaster monitoring and mitigation using aerospace technologies and integrated telecommunication networks", IEEE Aerospace and Electronic Systems Magazine, 23(4), April 2008.

[3] I. Oka and M.P.C. Fossorier, "A General Orthogonal Modulation Model for Software Radio", IEEE Transactions Communications, vol. 54, No.1, Jan. 2006, pp. 7-12.

[4] E. Marpanaji et al., "Experimental Study of DQPSK Modulation on SDR Platform", ITB Journal of Information and Communication Technology, vol. 1, No.2, 2007, pp. 84-98.

[5] GNU Radio. http://gnuradio.org. - Accessed on March. 2, 2013.

[6] Ettus Research LLC. http://www.ettus.com - Accessed on March. 2, 2013.

[7] C. Costanzo, V. Loscrí, E. Natalizio, T. Razafindralambo, "Nodes selfdeployment for coverage maximization in mobile robot networks using an evolving neural network", Computer Communications, 35(9), 2012.

[8] G. Molina and E. Alba and E. Talbi, "Optimal Sensor Network Layout Using Multi-Objective Metaheuristics", Journal of Universal Computer Science, n 15, pp 2549-2565, vol. 14, 2008.

[9] R. Surace, V. Loscrí, and E. Natalizio, "On the impact of the propagation environment on controlled mobility algorithms", in International Workshop on Mobility and Communication for Cooperation and Coordination (MC3) at IEEE ICNC 2012, pp. 62-66, 2012.

[10] S. Butman, B. Levitt, I. Bar-David, R. Lyon and M. Klass, "Design Criteria for Noncoherent Gaussian Channels with MFSK Signaling and Coding”, IEEE Transactions on Communications, vol. 24, No. 10, 1976 , pp. 1078-1088.

[11] F.M. Costa and H. Ochiai, "A comparison of modulations for energy optimization in wireless sensor network links", in IEEE Global Telecommunications Conference (GLOBECOM), pp 1-5, December 2010.

[12] S. Cui, A.J. Goldsmith and A. Bahai, "Energy-constrained modulation optimization", IEEE Transactions on Wireless Communications, 4(5):2349-2360, September 2005.

[13] Roving Networks wireless for less WiFly GX 802.11G Super Module: RN-121, RN-122, RN-123, RN-125. http://www.rovingnetworks.com 